\title{
Temporal Integration in Nasal Lateralization of Homologous Propionates
}

\author{
Paul M. Wise, Sean E. Toczydlowski, Kai Zhao, and Charles J. Wysocki \\ Monell Chemical Senses Center, 3500 Market Street, Philadelphia, Pennsylvania, 19104-3308
}

\begin{abstract}
For nasal irritation from volatile chemicals, a version of Haber's rule $\left(\mathrm{k}=\mathrm{C}^{\mathrm{n}} \mathrm{T}\right)$ can model the tradeoff between concentration (C) and duration of exposure (T) to achieve a fixed sensory impact, e.g., threshold-level irritation or a fixed supra-threshold intensity. The term " $\mathrm{k}$ " is a constant. The exponent, $\mathrm{n}$, represents how well the system integrates over time. An exponent of 1 indicates complete temporal integration: an $\mathrm{x}$-fold increase in stimulus-duration exactly compensates for cutting concentration 1/x. An exponent greater than 1 indicates incomplete temporal integration: more than an $\mathrm{x}$-fold increase in duration is needed. In a previous study of homologous alcohols, $\mathrm{n}$ varied systematically with number of methylene units: integration became more compete as the length of the carbon chain increased. To explore the generality of this finding, we tested homologous esters that differ in number of methylene units: n-ethyl propionate, n-propyl propionate, and n-butyl propionate. Nasal lateralization was used to measure irritation thresholds. Human subjects received a fixed concentration of a single compound within each experimenal session. Stimulus-duration was varied to find the briefest stimulus that caused lateralizable irritation. Concentration and compound varied across sessions. Consistent with results with $\mathrm{n}$-alcohols, integration became more complete as the number of methylene units increased. Lipid solubility varies with chain-length; hence solubility in the nasal mucosa may play a role in the dynamics of irritation. Further, preliminary analyses suggest that, for data pooled across both chemical series, $\mathrm{n}$ varied systematically with molecular parameters related to solubility and diffusion.
\end{abstract}

\section{Keywords}

Chemical irritation; pungency; chemesthesis; volatile organic compound; inhalation toxicology

\section{Introduction}

Vapors and gasses can trigger somatosensory perceptions that range from innocuous warming and cooling to burning and stinging. Mucus membranes, which offer relatively easy access to sensory nerve endings, prove most sensitive (Bryant and Silver, 2000; Doty and ComettoMuñiz, 2003; Doty et al., 2004). Chemical feel, or chemesthesis, in the eyes and upper airways is a common complaint in many problematic environments, and can have significant economic impact through lost productivity (CDC, 1991; Dalton, 2001; Fisk et al., 1993; Fisk \& Rosenfeld, 1997). Accordingly, government regulators set many occupational exposure-limits based on chemical irritation (Cain, 1996; NIOSH, 1994); however, basic data that relate stimulus to sensation remain limited, especially in humans.

Correspondence to: Dr. Paul M. Wise, Monell Chemical Senses Center, 3500 Market Street, Philadelphia, PA 19104-3308, Phone: 267-519-4799, FAX: 215-898-2084, email: pwise@monell.org. 
Data on the effects of duration of exposure are particularly limited. Rated irritation from suprathreshold, i.e., clearly perceptible, stimuli may grow with stimulus-duration over seconds or even minutes, though irritation may plateau and eventually fade as adaptation occurs (Anton et al., 1992; Cain et al., 1986; Frasnelli et al., 2003; Hempel-Jorgensen et al., 1999; Wise et al., 2003; Shusterman et al., 2003; Cain et al., 2008). A full understanding of these phenomena will require systematic research.

A few studies, mostly using brief (up to about 10 second) intra-nasal exposures, have systematically investigated temporal integration, i.e., the trade-off between concentration and duration of exposure (Shusterman et al., 2006). A simple model describes short-term temporal integration in nasal chemesthesis very well: a fixed-ratio increase in stimulus-duration can compensate for a fixed-ratio decrease concentration to maintain a fixed sensory impact, i.e., a just-detectable sensation (Wise et al., 2004; 2005; 2006; 2007) or a given supra-threshold intensity (Cometto-Muñiz \& Cain, 1984; Wise et al., 2005). This has held true for carbon dioxide, which stimulates through acidification of tissue (Shusterman and Avila, 2003), the base ammonia, and three, homologous n-alcohols (Wise et al., 2004; 2005; 2006; 2007). These studies suggest that sensory impact is related in some fashion to total mass delivered to the nose, i.e., some function of concentration (C) multiplied by time (T).

Haber's rule, viz. $\mathrm{C} * \mathrm{~T}=$ constant for a fixed endpoint, is perhaps the simplest mass integration model (Miller et al., 2000; Shusterman et al., 2006). If Haber's rule applies, time-concentration trading is perfect: e.g., a two-fold increase in stimulus-duration will exactly compensate for cutting concentration in half. However, in recent studies of detection of nasal irritation, integration ranged from slightly imperfect for ammonia (a 2.5 fold increase in duration compensated for cutting concentration in half) to highly imperfect for ethanol (a 5.8 fold increase in duration compensated for cutting concentration in half) (Wise et al., 2004; 2005; 2006; 2007). An exponentiated version of Haber's rule, $C^{n} T=$ constant, models these data very well. The exponent $n$ indicates degree of integration (see Data Analysis for more details) (Miller et al., 2000; Shusterman et al., 2006).

That integration tends to be imperfect, and varies across compounds, has immediate implications for studies of irritation. A growing body of literature on structure-activity relationships in irritant potency is based mostly on brief exposures in the laboratory (Abraham et al., 2001; 2003; Cain et al., 2006; Doty and Cometto-Muñiz, 2003; Hau et al., 1999). Often, subjects take one or more sniffs, and duration of exposure is not precisely controlled. Even if stimulus-duration is fixed using appropriate apparatus (e.g., an olfactometer), the observed structure-activity relationships could vary based on the duration of exposure if compounds differ in degree of integration. Ideally, studies would include multiple stimulus durations. The exponentiated version of Haber's rule could be a powerful tool in this effort, allowing researchers to characterize entire time-concentration trading functions by studying a few, carefully selected, combinations of concentration and duration. However, the degree of integration for any particular compound must be determined empirically.

Eventually, it would be desirable to move beyond the descriptive model outlined above to predict degree of integration based on molecular properties, both to interpret data collected with differing methods and to gain insights into underlying mechanisms. Wise and colleagues began with the hypothesis that more lipophillic molecules might accumulate better in the lipidrich nasal mucosa over time, and therefore demonstrate integration that is closer to perfect (Wise et al., 2007). Consistent with this hypothesis, degree of integration increased monotonically as number of methylene units (and lipid solubility) increased within a series of homologous n-alcohols. The current study explores the generality of this finding, and expands the data-base on structure-activity relationships in the dynamics of chemesthesis by examining temporal integration in the detection of nasal irritation from homologous esters. In addition, 
we propose a new model of integration, based on diffusive transport of molecules into the nasal mucosa, which moves beyond pure description.

\section{Materials and Methods}

\section{Operational definition of irritation threshold}

Irritation thresholds were measured using nasal lateralization. Subjects simultaneously received chemical vapor in one nostril and clean air in the other nostril. Subjects had to decide which nostril received chemical vapor, guessing if uncertain. Humans lack the ability to lateralize odors, but we can lateralize chemicals that stimulate somatosensory nerves (Doty and Cometto-Muñiz, 2003; Wysocki and Wise, 2003). Lateralization is often used in place of simple detection tasks to measure thresholds for chemical feel in the nose. In detection, odor is a confound because subjects can smell most chemicals at concentrations too low to cause detectable irritation.

Results of lateralization studies agree well with nasal detection thresholds measured in subjects who lack a sense of smell, and relative values (across irritants) agree well with eye irritation thresholds (reviewed by Doty and Cometto-Muñiz, 2003). Further, human nasal lateralization is in good general agreement with ethmoid nerve recordings and changes in respiration in animal models regarding structure-activity relationships in irritant potency for non-reactive volatile organic compounds (Alarie et al., 1998; Bryant and Silver, 2000; Doty and ComettoMuñiz, 2003). In short, there is support for the validity of nasal lateralization as a measure of nasal trigeminal thresholds.

\section{Materials}

Subjects received n-ethyl propionate $\left(\mathrm{C}_{2}\right.$; CAS\# 105-37-3) at 530, 647, 799, 987, 225, and 1524 ppm, n-propyl propionate ( $\mathrm{C}_{3}$; $\left.\mathrm{CAS} \# 106-36-5\right)$ at 241, 319, 426, 564, 732, and $979 \mathrm{ppm}$, and n-butyl propionate $\left(\mathrm{C}_{4}\right.$; CAS\# 590-01-2) at $118,188,322,503$, and $833 \mathrm{ppm}$. These concentrations were target values. Actual concentrations deviated from these targets by less than five percent (see calibration). Pilot testing informed the choice of concentrations for each compound. The concentrations were selected to span a range from detectable with presentations of about 0.2 seconds to the lowest concentration that subjects could detect with pulses less than 10 seconds. Six concentrations were selected for both $C_{2}$ and $C_{3}$ because pilot tests were less conclusive regarding the lowest concentration for those compounds.

\section{Subjects}

A total of 22 ( 14 female) healthy, adult, non-smokers participated. Ages ranged from 18 to 56 (average $=31.5$ ). Subjects signed consent forms approved by the University of Pennsylvania Institutional Review Board before experiments began. All subjects were paid, except for author $\mathrm{PW}$. The author was blind to any conditions that could cue responses trial-by-trial, and his data resembled that of other subjects. Analyses that excluded the author's data supported the same conclusions. The experiment was planned as a complete within-subjects design, but changes in subject availability made this impossible to continue. Ten subjects contributed data for all three compounds, four subjects contributed data for two compounds, and eight subjects contributed data for only one compound. Subject-samples included 15 for both ethyl and propyl propionate, and 16 for butyl propionate.

\section{Apparatus}

The experiments used an air-dilution olfactometer, which other reports describe in greater detail (Wise et al., 2005; 2006; 2007). Briefly, room air was pumped through desiccant and a carbon filter, then warmed and re-humidified. Some of the treated flow passed through glass 
vessels containing pure, liquid propionate. This propionate-laden air was then diluted with additional treated air to form the desired stimulus-concentration (target to be lateralized). Solenoid valves could gate a stimulus (either propionate-laden target or a clean-air blank) to either nostril. Stimuli entered the nostrils at $5( \pm 0.05) \mathrm{L} / \mathrm{min} ., 37( \pm 0.5)^{\circ} \mathrm{C}$, and $90( \pm 3) \%$ RH. Between stimulus-presentations, the olfactometer produced a steady stream of "background" air with the same flow-rate, temperature, and humidity as the stimuli. The olfactometer switched between stimuli and background flow rapidly, with minimal changes in pressure, thereby allowing a focus on chemical stimulation of somatosensory nerves. Despite tight control over timing, the device could present only a single concentration without manual adjustment and calibration, so only stimulus-duration was manipulated within an experimental session (see Procedure). Further, the device was configured to present only a single compound at a time, which reduced the chances of cross-contamination of stimuli, but imposed a limitation on the design (see Procedure and the Limitations section of the discussion).

\section{Stimulus presentation}

Subjects practiced velopharyngeal closure while they sampled stimuli. This breathing technique, in which subjects isolate the nasal cavity from the rest of the airways using the soft palate, prevents fluctuations in nasal air-flow from respiration (Kobal and Hummel, 1991). Air flowed from the olfactometer into the nose through flexible Tygon ${ }^{\circledR}$ tubing $(4 \mathrm{~mm}$ outer diameter). The tubing extended about $0.75 \mathrm{~cm}$ into subjects' nostrils, and flow exited the nostrils around the tubes.

\section{Calibration}

All calibration was performed on air-flow at the output of the olfactometer. Flow-rate was checked using a Gillibrator 2 flow-meter (Gillian Instrument Corp.; Wayne, NJ), humidity was checked using a Digitron 2020R hygrometer (Topac Instruments; Hingham, MA), and temperature was checked using a BAT-12 thermocouple reader (Physiotemp Instruments; Clifton, NJ). Experimenters verified that minimal changes in flow occurred when switching from background air to stimuli using a fast-response pressure-transducer (CyQ line, custom made; Cybersense; Nicholasville, KY). Each day, experimenters adjusted the vapor-phase concentration of the propionate to be studied to the desired ppm value using a MiniRAE 2000 photo-ionization detector (RAE Systems; Sunnyvale, CA). Calibration curves for the photoionization detector were created using gas-phase standards. The standards were created by injecting known masses of each propionate into air-filled Tedlar® gas sampling bags.

\section{Procedure}

Subjects placed the tubes in their nostrils at the start of each trial, and established velopharyngeal closure. Next, subjects began a 10 second countdown by clicking a mouse. Beeps accompanied the last three seconds of the countdown. After the countdown, the computer presented a variable-duration stimulus. The nostril that received the target was selected randomly, trial by trial. Subjects remained in position for three seconds after the stimulus ended, then clicked a virtual button on a monitor to record which nostril received the target. Forty-five seconds after subjects entered a response, a prompt appeared on the monitor to begin the next trial. In total, more than 55 seconds elapsed between stimulus-presentations.

During a given experimental session, subjects received a fixed concentration of a single propionate. Stimulus-duration varied to find the briefest pulse that subjects could reliably lateralize. A forced-choice, ascending method of limits was used (Cain et al., 1988; Wise et al., 2006). Pilot work identified durations that were at least three, $0.10 \log _{10}$ duration-steps below threshold for most subjects. Duration was increased by one step after an incorrect response. Duration remained the same after a correct response. Five consecutive correct responses at a given concentration ended a run, and the concentration at which this occurred 
served as the threshold estimate. In the event of five correct responses at the starting duration (a rare occurrence), the experimenter cut the starting duration in half and began again. This method, which was validated against a more intensive threshold-method in earlier work (Wise et al, 2006; Wise et al., 2008), allowed reasonably rapid data-collection. Most Runs required about 10-15 minutes. After a rest period of at least five minutes, subjects completed a second run using the same concentration, compound, and procedure.

Most subjects had served in previous studies using the same methods. Nevertheless, all subjects completed one practice session for each compound they received, using an intermediate concentration. After the practice session (on subsequent days), subjects completed a single session for each concentration, in irregular order. Subjects were limited to a single session on a given day, and completed all sessions for a given compound within five weeks. Because of the need to thoroughly clean the olfactometer between compounds, and to replace some tubing in the process, only one compound could be tested at a time. The compounds were tested in the following (randomly-selected) order: ethyl propionate, butyl propionate, propyl propionate.

\section{Data analysis}

In an imperfect integration model (exponentiated version of Haber's rule described in the Introduction), solving for $\mathrm{T}$ yields: $\mathrm{T}=\mathrm{kC}^{-\mathrm{n}}$. Because this equation is a power function, plots of $\log$ duration vs. $\log$ concentration can be fit with a simple linear equation:

$$
\log (\mathrm{T})=-\mathrm{n}[\log (\mathrm{C})]+\mathrm{k}^{\prime}
$$

" $T$ " represents the threshold stimulus-duration required for lateralization. "C" represents concentration, and $\mathrm{k}$ ' is a constant ( $\log$ of $\mathrm{K})$. A slope, $\mathrm{n}$, of -1 indicates perfect integration, or perfect time-concentration trading. In this transformation of the model, a slope less than -1 indicates imperfect integration, and a slope of greater than -1 indicates greater than perfect integration.

To analyze group functions, the two threshold estimates for each concentration of each propionate were averaged within subjects, then thresholds for each concentration of each propionate were averaged across subjects. The linear equation was fit, using least-squares regression, to resulting functions of log threshold duration (Y-axis) versus log concentration (X-axis). Regression generated a 95\% confidence interval for the slopes of each compound. Two slopes were judged to be different if the CI of each compound excluded the mean slope of the other. This standard for significance roughly corresponds to a series of t-tests with an alpha level of 0.05 (two-tailed), i.e., that the means differ by at least two standard errors (adjusted for sample size).

In a second analysis, functions were fit to data from individual subjects. We did not collect sufficient data from individuals for detailed analysis. Previous work did test individuals intensively enough to ensure that the exponentiated version of Haber's rule provided a good description of time-concentration trading functions for individuals (Wise et. al., 2004; 2005), and that extensive testing of a small number of subjects yielded essentially the same results as more rapid testing of a larger sample (Wise et al., 2006). Nevertheless, an analysis of fits to individual integration functions qualifies as another valid approach, and therefore complements analysis of group functions. A slope was computed for each stimulus and subject. Slopes were submitted to a one-way, between-subjects, analysis of variance (ANOVA) to test the overall effect of number of methylene units within the family of propionates. Differences in slope between individual propionates were assessed using independent-samples t-tests (with a Bonferroni correction for multiple comparisons). In addition, fitted slopes from the 10 subjects who received all three compounds were submitted to a one-way, within-subjects ANOVA, 
with follow-up analyses conducted using Bonferroni-corrected, matched-pairs t-tests. However, the results of these analyses are not presented since they support exactly the same conclusions as the between-subjects analysis on all subjects.

In a third set of analyses, we combined integration slopes for propionates with similar data on n-alcohols (Wise et al., 2007) to examine correlations between molecular properties and integration across chemical families. One property, lipophilicity, was expressed as log octanol/ water partition coefficient $(\log \mathrm{P})$. For a two-phase system, $\log \mathrm{P}$ increases with the proportion of molecules in the octanol phase at equilibrium (lipophilicity increases with $\log \mathrm{P}$ ). In addition, the logarithm of Henry's law constant $(\log \mathrm{H})$, provided an analogous value for partitioning between air and water, as might happen when molecules partition from the vapor phase into the watery, upper layer of mucus. Finally, we examined the log of diffusivity, which relates to how quickly molecules diffuse through water $(\log D)$. Diffusivity in lipids would be conceptually relevant, but the relative diffusivities of the various compounds should be comparable to those in water. Pearson's correlation coefficients were used assess how integration slope changed with each parameter. In addition, multiple regression was used to determine how well integration slope could be predicted from combinations of molecular parameters. We have integration data on only six compounds, viz. three propionates and three alcohols, hence the analyses lack sufficient power to reach firm conclusions and should be regarded as preliminary.

\section{Results}

\section{Analysis of group functions}

Reliable lateralization failed at lower concentrations for some subjects, even with stimuli as long as 10 seconds. For ethyl propionate, two subjects failed at the lowest concentration, and two failed at the second lowest concentration. For propyl propionate, four failed at the lowest concentration and two failed at the second lowest concentration. For butyl propionate, three failed at the lowest concentration and one failed and the second lowest concentration. For each compound, at least one subject lateralized the lowest concentration, but failed to lateralize the second lowest concentration, suggesting possible within-subjects fluctuation in sensitivity across days.

Regardless of these considerations, for all three compounds, subjects were able to lateralize increasingly weaker concentrations if stimulus-duration increased (Figure 1). Linear functions fit group data reasonably well, accounting for $99 \%$ of the variance in thresholds for ethyl propionate, $96 \%$ of the variance for propyl propionate, and $98 \%$ of the variance for butyl propionate. Slopes of best-fit linear functions, with $95 \%$ CIs in parentheses, were: $-2.65(-3.11$ to -2.20$)$ for ethyl propionate, $-1.87(-2.39$ to -1.35$)$ for propyl propionate, and $-1.04(-1.32$ to -.77 ) for butyl propionate. Accordingly, to compensate for a two-fold decrease in concentration, one would need to increase duration by about 6.3 fold for ethyl propionate, about 3.6 fold for propyl propionate, and about 2.06 fold for butyl propionate (essentially perfect integration). By the criterion that the $95 \%$ CIs should not include means for other compounds, slopes increased monotonically with number of methylene units.

\section{Analysis of slopes from individual subjects}

Linear fits to data for individual subjects (see Figure 2 for functions for three representative individuals) accounted for an average of $82 \%$ of the variance in thresholds for ethyl propionate, $77 \%$ of the variance in thresholds for propyl propionate, and $78 \%$ of the variance in thresholds for butyl propionate. Average slopes, with standard deviations in parentheses, for the three compounds were -2.61 (1.05) for ethyl propionate, -2.03 (0.43) for propyl propionate and 
-1.14 (0.44) for butyl propionate. Accordingly, slopes from analysis of individual data agree reasonably well with those from analysis of group data.

In a one-way ANOVA, the effect of compound reached significance, $F(2,43)=17.63$, $p<$ 0.001. Pair-wise tests revealed a marginal difference between ethyl propionate and propyl propionate, $\mathrm{t}(28)=1.97, \mathrm{p}=0.06$ (two-tailed), a significant difference between ethyl propionate and butyl propionate, $\mathrm{t}(29)=5.16, \mathrm{p}<0.001$, and a significant difference between propyl propionate and butyl propionate, $\mathrm{t}(29)=5.72, \mathrm{p}<0.001$.

\section{Preliminary analyses of the relationship between molecular parameters and integration across propionates and alcohols}

Correlation coefficients between integration slope and various combinations of molecular parameters appear in Table 1. With only six data points, very few results reach statistical significance. This is especially true for combinations of molecular parameters, since each variable entered into the regression consumes an additional degree of freedom. Still, some degree of association seems likely, especially for $\log D$ and $\log P$. Further, examination of scatter-plots (Figure 3) reveals that the propionates and alcohols appear to follow separate trends, with a strong linear association between molecular parameters and integration slope for each chemical family. Accordingly, the importance of partitioning between air and water $(\log \mathrm{H})$ cannot be discounted at this time, despite the low correlation for data pooled across chemical families. Interestingly, if one plots the observed integration slope vs. predicted slope based on the optimal combination of all three molecular properties (Figure 4), regression lines fit to data from the two chemical families coincide. Across chemical families, the optimal combination of three variables predicts 92 percent of the variance in observed integration slopes.

\section{Discussion and Conclusions}

\section{A model of short-term integration}

An exponentiated version of Haber's rule (see Introduction) described short-term integration in nasal lateralization well for $\mathrm{CO}_{2}$, ammonia, and three n-alcohols (Wise et al., 2004; 2005; 2006; 2007). The reasonable linear fits in Figure 1 show that this model also described lateralization for homologous esters, thereby extending past results. Considering similar findings on ratings of supra-threshold irritation (Cometto-Muñiz and Cain, 1984; Shusterman et al., 2006; Wise et al., 2005), it seems that the exponentiated version of Haber's rule might well apply in a wide variety of situations.

The model is descriptive. Researchers must characterize time-concentration trading functions for individual compounds empirically. However, until we can predict slopes of integration functions based on molecular properties, the model can help characterize entire timeconcentration trading functions based on measurements of a few, carefully selected points.

\section{Differences among compounds in degree of integration}

Though the same general model describes time-concentration trading for various compounds, chemicals vary in degree of integration (see Introduction). Consistent with the hypothesis that more lipophilic molecules accumulate more readily in the peri-receptor environment, and thus integrate better over time, slopes of time-concentration trading functions fell monotonically with number of methylene units within a series of aliphatic alcohols (Wise et al., 2007). This pattern generalized to homologous esters in the current study. Analyses of data pooled across aliphatic series found an association between integration slope and lipid solubility (Table 1), though it was also clear that no single molecular parameter could account for all differences in slope (Figure 3). A preliminary analysis found that a combination of lipid solubility, water 
solubility, and diffusivity could account for most of the variance in integration slopes (Figure 4). This result is consistent with the hypothesis that integration is related to transport from the vapor phase into the nasal mucosa, though firm conclusions would require data on a larger set of compounds.

The current results also extend the literature on chemesthetic thresholds measured using more traditional paradigms. For many non-reactive volatile organic compounds, irritation threshold decreases as lipid-solubility increases (Abraham et al., 2001; 2003; Cain et al., 2006; Doty and Cometto-Muñiz, 2003)..Most studies in this area employ brief presentations, often for uncontrolled durations, e.g., a single sniff, or a single, fixed duration using an olfactometer. Since the slopes of integration functions vary systematically with physico-chemical parameters, sampling at a fixed duration would provide an incomplete picture of structureactivity relationships. An ideal protocol would include multiple durations.

Possible mechanisms-The exponentiated version of Haber's rule is a black box that covers all events from entry of the stimulus into the nares to execution of the behavioral response. We have discussed the possibility that integration occurs through accumulation of molecules in the nasal mucosa over time. Concurrent loss of molecules, e.g., by diffusion into the sub-mucosal bloodstream, muco-cilliary clearance, or through enzymatic action, might contribute to differences among molecules in degree of integration. Other possibilities include central or peripheral neural integration, with adaptation tending to cause imperfect integration, and progressive recruitment of different types of fibers, with imperfect integration occurring as the population of un-stimulated fibers diminishes. These possibilities are neither exhaustive nor exclusive. As discussed elsewhere (Cain, 1990; Wise et al., 2004), a detailed understanding of underlying mechanisms will require an inter-disciplinary effort that combines behavioral work, studies of stimulus-dynamics in the nasal mucosa, and physiological recordings at various levels in the nervous system.

\section{A diffusive transport model of integration}

To help guide further behavioral research, we have developed the transport hypothesis outlined above into a more formal model (Appendix). According to the model, 1) detectable irritation occurs when concentration in the mucosa reaches a critical level, 2) rate of diffusion of molecules into the mucosa from the vapor-phase decreases according to a compressive function as mucosal concentration increases, and 3) leakage through diffusion of molecules from the mucosa into the sub-mucosal blood-stream increases as a function of mucosal concentration. The balance of these processes determines degree of integration. This model fit integration functions for both the aliphatic alcohols (Wise et al., 2007) and propionates (current report) reasonably well (Figure 5). Arbitrary perturbations of parameters suggest that rate of submucosal blood flow does not contribute much to differences in integration slope between compounds, i.e., does not change model fit significantly. On the other hand, rate of diffusion in the mucosa did influence model fit, suggesting that this may be an important factor in integration.

Unfortunately, current data do not allow a rigorous test of the model. In part, this is because predicted deviations from linearity only occur for stimuli too brief (less than $200 \mathrm{~ms}$ ) to reliably manipulate with our apparatus (less than $200 \mathrm{~ms}$ ). However, the model can also predict integration functions for non-uniform stimuli, e.g., stimuli that turn on and off repeatedly during the course of presentation. This more rigorous test of the diffusive transport model might suggest that basic transport factors are insufficient to explain integration. 


\section{Limitations}

1) Stimuli were injected into the nose while subjects practiced velopharyngeal closure. This method allowed good stimulus-control, but patterns of flow in the nose probably differed from those of natural breathing, which could in turn affect deposition and absorption of compounds in the nasal mucosa (e.g., Frederick et al., 1994, 1998; Kurtz et al., 2004; Morris, 2001). Studies using natural breathing would complement the current findings. 2) Stimuli were run in a fixed order for all subjects, since it took days to clean the olfactometer between compounds. Previous experiments showed that slopes of integration functions for a model compound replicate quite well across experiments, even when psychophsycial methods vary (Wise et al., 2006; 2007), but we cannot completely rule out order effects. 3) Integration was studied over less than 10 seconds. Additional studies will be required to determine how these data relate to longer exposures under more natural conditions. 4) Finally, we studied only young, healthy nonsmokers, whose responses may differ compared to other populations (Shusterman, 2002). It might prove interesting to determine whether patient populations, e.g., those who suffer from chronic rhinitis, differ from healthy controls with respect to temporal integration.

\section{Conclusions}

Integration improved monotonically with number of methylene units within an aliphatic series, which extends a previous study on aliphatic alcohols, and suggests that at least some aspects of sensory dynamics can be predicted based on molecular parameters. Further, integration tends to be more complete as lipid solubility increases, which is consistent with the hypothesis that transport from the vapor phase to the nasal mucosa plays a role in the dynamics of sensation. Thus far, a structure-activity approach to understanding the dynamics of nasal chemesthesis shows promise.

\section{Acknowledgements}

This work was supported in part by Kraft Foods through the Term Chair in Chemosensory Psychophysics at Monell (PMW), NIEHS grant 5R03ES013969 (PMW) and the Monell-Jefferson Chemosensory Clinical Research Center grant from NIDCD, 5P50DC006760, which supports the research of KZ..

\section{References}

Abraham MH, Gola JMR, Cometto-Muñiz JE, Cain WS. The correlation and prediction of VOC thresholds for nasal pungency, eye irritation and odour in humans. Indoor-Built Environ 2001;10(34):252-257.

Abraham MH, Hassanisadi M, Jalali-Heravi M, Ghafourian T, Cain WS, Cometto-Muñiz JE. Draize rabbit eye test compatibility with eye irritation thresholds in humans: A quantitative structure-activity relationship analysis. Toxicol. Sci 2003;76(2):384-391. [PubMed: 14514959]

Anton F, Euchner I, Handwerker HO. Psychophysical examination of pain induced by defined $\mathrm{CO}_{2}$ pulses applied to the nasal mucosa. Pain 1992;49(1):53-60. [PubMed: 1594282]

Alarie Y, Schaper M, Nielsen GD, Abraham MH. Structure-activity relationships of volatile organic chemicals as sensory irritants. Arch. Toxicol 1998;72:125-140. [PubMed: 9520136]

Bryant, BP.; Silver, WL. Chemesthesis: The common chemical sense. In: Finger, TE.; Silver, WL.; Restrepo, D., editors. The Neurobiology of Taste and Smell. Vol. 2nd ed.. New York: Wiley-Liss; 2000. p. 73-100.

Cain, WS. Perceptual characteristics of nasal irritation. In: Green, BG.; Mason, JR.; Kare, MR., editors. Chemical Senses (Vol. 2): Irritation. Marcel Decker; New York: 1990. p. 43-58.

Cain, WS. Overview: Odors and irritation in indoor pollution. In: Gammage, RB.; Berven, BA., editors. Indoor Air and Human Health. Vol. 2nd ed.. Lewis; Boca Raton: 1996. p. 23-30.

Cain WS, Gent JF, Goodspeed RB, Leonard G. Evaluation of olfactory dysfunction in the Connecticut Chemosensory Clinical Research Center. Laryngoscope 1988;98(1):83-88. [PubMed: 3336267] 
Cain WS, Jalowayski AA, Kleinman M, Lee NS, Lee BR, Ahn BH, Magruder K, Schmidt R, Hillen BK, Warren CB, Culver BD. Sensory and associated reactions to mineral dusts: Sodium borate, calcium oxide, and calcium sulfate. J. Occup. Environ. Hyg 2004;1(4):222-236. [PubMed: 15204861]

Cain WS, Jalowayski AA, Schmidt R, Kleinman M, Magruder K, Lee KC, Culver BD. Chemesthetic responses to airborne mineral dusts: boric acid compared to alkaline materials. Int. Arch. Occup. Environ. Health 2008;81(3):337-345. [PubMed: 17609973]

Cain WS, Lee NS, Wise PM, Schmidt R, Ahn BH, Cometto-Muñiz JE, Abraham MH. Chemesthesis from volatile organic compounds: Psychophysical and neural responses. Physiol. Behav 2006;88(45):317-324. [PubMed: 16806320]

Cain, WS.; See, LC.; Tosun, T. IAQ '86: Managing the Indoor Air for Health and Energy Conservation. Atlanta: ASHRAE; 1986. Irritation and odor from formaldehyde: Chamber studies; p. 126-137.

Centers for Disease Control. Discomfort from environmental tobacco smoke among employees at worksites with minimal smoking restrictions-United States, 1988. Morbid, Mortal. Weekly Rep 1991;41:351-354.

Cometto-Muñiz JE, Cain WS. Temporal integration of pungency. Chem. Senses 1984;8(4):315-327.

Cometto-Muñiz JE, Cain WS, Abraham MH, Sanchez-Moreno R. Cutoff in detection of eye irritation from vapors of homologous carboxylic acids and aliphatic aldehydes. Neuroscience 2007;145(3): 1130-1137. [PubMed: 17270354]

Dalton P. Evaluating the human response to sensory irritation: Implications for setting occupational exposure limits. AIHA J 2001;62(6):723-729.

Dalton P, Wysocki CJ, Brody MJ, Lawley HJ. The influence of cognitive bias on the perceived odor, irritation and health symptoms from chemical exposure. Int. Arch. Occup. Environ. Health 1997;69 (6):407-417. [PubMed: 9215927]

Doty, RL.; Cometto-Muñiz, JE. Trigeminal chemosensation. In: Doty, RL., editor. Handbook of Olfaction and Gustation. Vol. 2nd ed.. New York: Marcell Decker; 2003. p. 981-999.

Doty RL, Cometto-Muñiz JE, Jalowayski AA, Dalton P, Kendal-Reed M, Hodgson M. Assessment of upper respiratory tract and ocular irritative effects of volatile chemicals in humans. Crit. Rev. Toxicol 2004;34(2):85-142. [PubMed: 15112751]

Frasnelli J, Lötsch J, Hummel T. Event-related potentials to intranasal trigeminal stimuli change in relation to stimulus concentration and stimulus duration. J. Clin. Neurophysiol 2003;20(1):80-86. [PubMed: 12684563]

Frederick CB, Morris JB, Kimbell JS, Morgan KT, Scherer PT. Comparison of four biologically based dosimetry models for the deposition of rapidly metabolized vapors in the rodent nasal cavity. Inhal. Toxicol 1994;6(Suppl):135-157.

Frederick CB, Bush ML, Lomax LG, Black KA, Finch L, Kimbell JS, Morgan KT, Subramaniam RP, Morris JB, Ultman JS. Application of a hybrid computational fluid dynamics and physiologically based inhalation model for interspecies dosimetry extrapolation of acidic vapors in the upper airways. Toxicol. Appl. Pharmacol 1998;152(1):211-231. [PubMed: 9772217]

Fisk WJ, Rosenfeld AH. Estimates of improved productivity and health from better indoor environments. Indoor Air 1997;7:158-172.

Fisk WJ, Mendell MJ, Daisey JM, Faulkner D, Hodgson AT, Nematollahi M, Macher JM. Phase 1 of the California Health Building Study: A summary. Indoor Air 1993;3:246-254.

Garner WR, Miller GA. The masked threshold of pure tones as a function of duration. J. Exp. Psychol 1947;37(4):293-305.

Green, BG.; Mason, JR.; Kare, MR. Preface. In: Green, BG.; Mason, JR.; Kare, MR., editors. Chemical Senses (Vol. 2): Irritation. New York: Marcel Decker; 1990. p. v-vii.

Hansch, C.; Leo, A.; Hoekman, D. American Chemical Society. Washington, D.C.: 1995. Exploring QSAR-Hydrophobic, Electronic and Steric Constraints.

Hau KM, Connell DW, Richardson BJ. Quantitative structure-activity relationships for nasal pungency thresholds of volatile organic compounds. Toxicol. Sci 1999;47(1):93-98. [PubMed: 10048157]

Hempel-Jorgensen A, Kjaergaard SK, Molhave L, Hudnell HK. Time course of sensory eye irritation in humans exposed to n-butanol and 1-octene. Arch. Environ. Health 1999;54(2):86-94. [PubMed: 10094285] 
Howard, PH.; Meylan, WM.; Fake, FF. Handbook of physical properties of organic chemicals. 1st E. CRC Press/Lewis Publishers; Boca Raton, FL: 1997.

Kasanen JP, Pasanen AL, Pasanen P, Liesivuori J, Kosma VM, Alarie Y. Stereospecificity of the sensory irritation receptor for nonreactive chemicals illustrated by pinene enantiomers. Arch. Toxicol 1998;72(8):514-523. [PubMed: 9765067]

Kobal, G.; Hummel, T. Olfactory evoked potentials in humans. In: Getchell, TV.; Doty, RL.; Bartoshuk, L.; Snow, JB., editors. Smell and Taste in Health and Disease. New York: Raven Press; 1991. p. 255-275.

Kurtz DB, Zhao K, Hornung DE, Scherer P. Experimental and numerical determination of odorant solubility in nasal and olfactory mucosa. Chem. Senses 2004;29(9):763-773. [PubMed: 15574812]

Meylan WM, Howard PH. Atom/fragment contribution method for estimating octanol-water partition coefficients. J. Pharmaceut. Sci 1995;84(1):83-92.

Miller FJ, Schlosser PM, Janszen DB. Haber's rule: a special case in a family of curves relating concentration and duration of exposure to a fixed level of response for a given endpoint. Toxicology 2000;149(1):21-34. [PubMed: 10963858]

Morris JB. Overview of upper respiratory tract vapor uptake studies. Inhal. Toxicol 2001;13(1):335-345. [PubMed: 11295866]

NIOSH. NIOSH pocket guide to chemical hazards. National Institute for Occupational Safety and Health; Cincinnati: 1994. (Publication No. 94-116)

Shusterman D. Individual factors in nasal chemesthesis. Chem. Senses 2002;27(6):551-564. [PubMed: 12142331]

Shusterman D, Avila PC. Real-time monitoring of nasal mucosal pH during carbon dioxide stimulation: Implications for stimulus dynamics. Chem. Senses 2003;28(7):595-601. [PubMed: 14578121]

Shusterman D, Matovinovic E, Salmon A. Does Haber's law apply to human sensory irritation? Inhal. Toxicol 2006;18(7):457-471. [PubMed: 16603477]

Thüerauf N, Kaegler M, Dietz R, Barocka A, Kobal G. Dose-dependent stereoselective activation of the trigeminal sensory system by nicotine in man. Psychopharmacol 1999;142(3):236-243.

Welty, JR.; Wicks, GE.; Wilson, RE. Fundamentals of Momentum, Heat and Mass Transfer. Vol. 2nd Edn.. Wiley; New York: 1976.

Wise PM, Canty TM, Wysocki CJ. Temporal integration of nasal irritation from ammonia at threshold and supra-threshold levels. Toxicol Sci 2005;87(1):223-231. [PubMed: 15976196]

Wise PM, Canty TM, Wysocki CJ. Temporal integration in nasal lateralization of ethanol. Chem. Senses 2006;31(3):227-235. [PubMed: 16384921]

Wise PM, Radil T, Wysocki CJ. Temporal integration in nasal lateralization and nasal detection of carbon dioxide. Chem. Senses 2004;29(6):137-142. [PubMed: 14977810]

Wise PM, Toczydlowski SE, Wysocki CJ. Temporal integration in nasal lateralization of homologous alcohols. Toxicol. Sci 2007;99(1):254-259. [PubMed: 17548891]

Wise PM, Wysocki CJ, Radil T. Time-intensity ratings of nasal irritation from carbon dioxide. Chem. Senses 2003;28(9):751-760. [PubMed: 14654442]

Wysocki, CJ.; Wise, P. Methods, approaches, and caveats for functionally evaluating olfaction and chemesthesis. In: Deibler, K.; Delwiche, JF., editors. Handbook of Flavor Characterization: Sensory, Chemical and Physiological. Marcel Dekker; New York: 2003. p. 1-40.

Zhang X, Zhang QY, Liu D, Su T, Weng Y, Ling G, Chen Y, Gu J, Schilling B, Ding X. Expression of cytochrome $\mathrm{p} 450$ and other biotransformation genes in fetal and adult human nasal mucosa. Drug Metab. Dispos 2005;33(1):1423-1428. [PubMed: 16014766]

\section{Appendix}

\section{Appendix}

The diffusive mucosal transport model, developed based on previous published studies (Kurtz et al., 2004, Zhao et al, 2004), takes the form of a partial differential equation: 


$$
\frac{\partial C}{\partial t}=D_{m} \frac{\partial^{2} C}{\partial y^{2}}+S(y, t)
$$

where $\mathrm{C}$, a function of $\mathrm{y}$ and $\mathrm{t}$, represents mucosal concentration, $\mathrm{D}_{\mathrm{m}}$ represents the binary diffusivity of the chemical in the mucosa, $y$ is the axis direction perpendicular to the mucosal surface ( 0 at the surface), and $\mathrm{S}(\mathrm{y}, \mathrm{t})$ is the source term for any increase or decrease of mucosal concentration due to metabolic or chemical reactions.

The initial condition for equation (1) is: $C(y, 0)=0$, i.e., 0 concentration prior to introducing the stimulus. The boundary condition at the mucosal surface $(\mathrm{y}=0)$ is defined as: $\mathrm{C}(0, \mathrm{t})=\mathrm{C}_{\mathrm{st}} \beta$, where $\mathrm{C}_{\mathrm{st}}$ is the air phase stimulus concentration and $\beta$ is the air/mucus partition coefficient (solubility) of the stimulus. At $\mathrm{y}=\mathrm{H}_{\mathrm{m}}$ (bottom interface of the mucosal layer; the thickness of which is $\mathrm{H}_{\mathrm{m}}$ ), we have, $D_{m} \frac{\partial C}{\partial y}=Q_{b} C \beta_{\text {blood }}$, a balance of flux. The term on the left of the equation is the one dimensional binary diffusive flux at the bottom of the mucosal layer. This term is balanced by flux carried away by sub-mucosal blood flow (with flow-rate of $\mathrm{Q}_{\mathrm{b}}$ ). $\beta_{\text {blood }}$ is the mucosa/blood partition coefficient.

Equation (1) was solved numerically in Matlab, which reveals a compressive increase in mucosal concentration over time for a given concentration. The model assumes that psychophysical irritation-threshold is reached at a fixed mucosal concentration, regardless of vapor-phase concentration. Integration often falls short of perfection because rate of chemical inflow is limited by diffusion and decreases as molecules accumulate in the mucosal layer. Thus far, we have fit the model to empirical time-concentration trading curves assuming no chemical or metabolic reactions (see Figure 3 ). For the parameter values, $D_{m}$ were initially set to their water values for the chemicals but allowed adjustment to reflect the distinctive property of the mucosa (Kurtz et al., 2004). Qb was set to a fixed value of $10^{-6} \mathrm{~mL} / \mathrm{s}$, a rough estimation of sub-mucosal blood perfusion rate. $\mathrm{H}_{\mathrm{m}}$ was set to $100 \mu \mathrm{m}$, a rough estimation of mucosal thickness. Goodness of fit to data was determined by standard nonlinear regression analysis (criterion of least square error). 


\section{0}

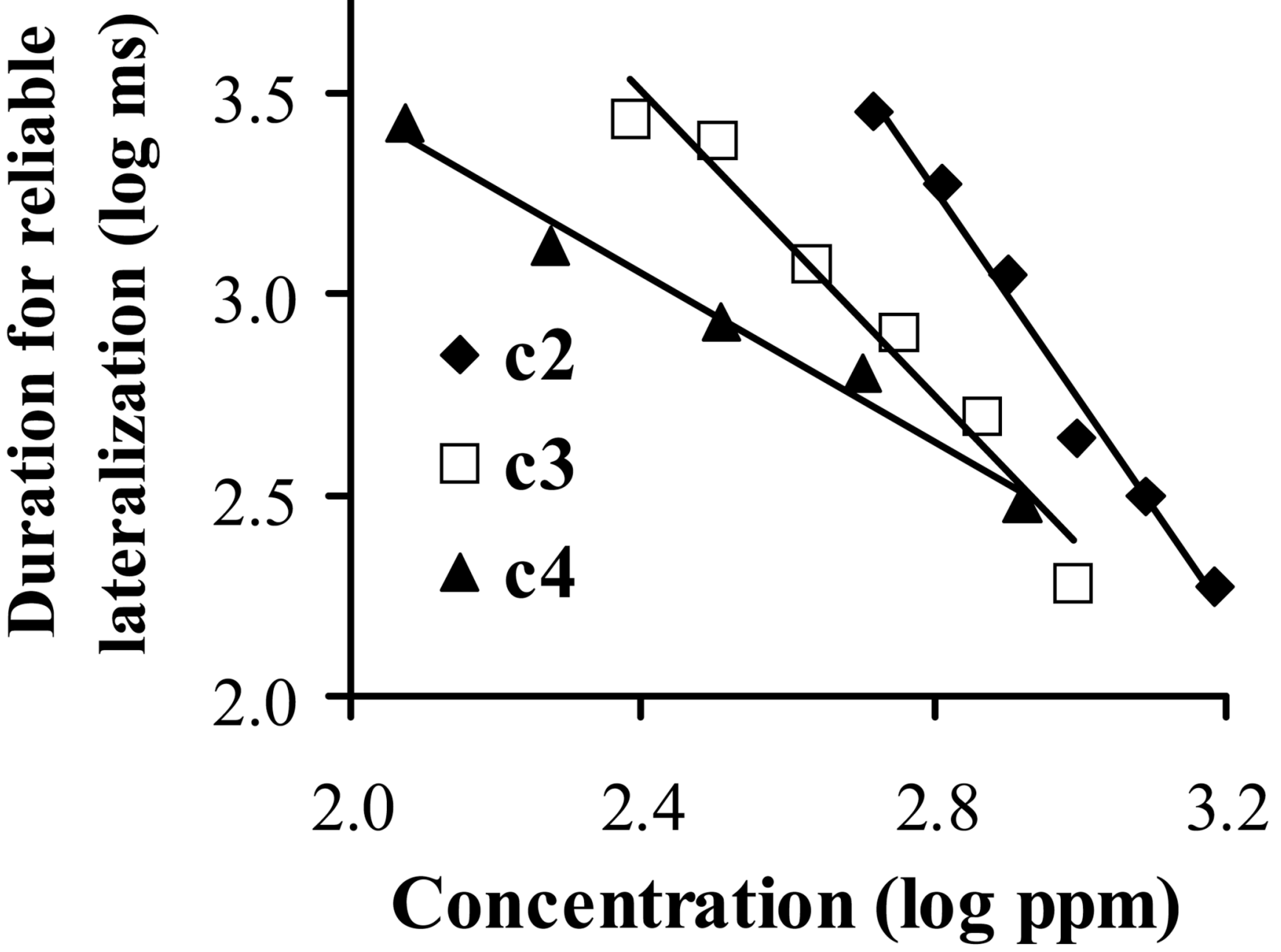

Fig. 1.

Threshold stimulus-duration (log ms) vs. concentration (log ppm) for ethyl propionate (filled diamonds), propyl propionate (open squares), and butyl propionate (filled triangles). Lines represent linear functions fit to data using least-squares regression. For ethyl propionate, the best fit equation was: $\log T=-2.65 \log C+10.40, R^{2}=.99$, where " $T$ " represents duration threshold (ms) and "C" represents concentration ( $\mathrm{ppm})$. The best fit equation for propyl propionate was: $\log \mathrm{T}=-1.87 \log \mathrm{C}+7.99, \mathrm{R}^{2}=.96$. For butyl propionate: $\log \mathrm{T}=-1.04 \mathrm{Log} \mathrm{C}$ $+5.55, \mathrm{R} 2=.98$. 


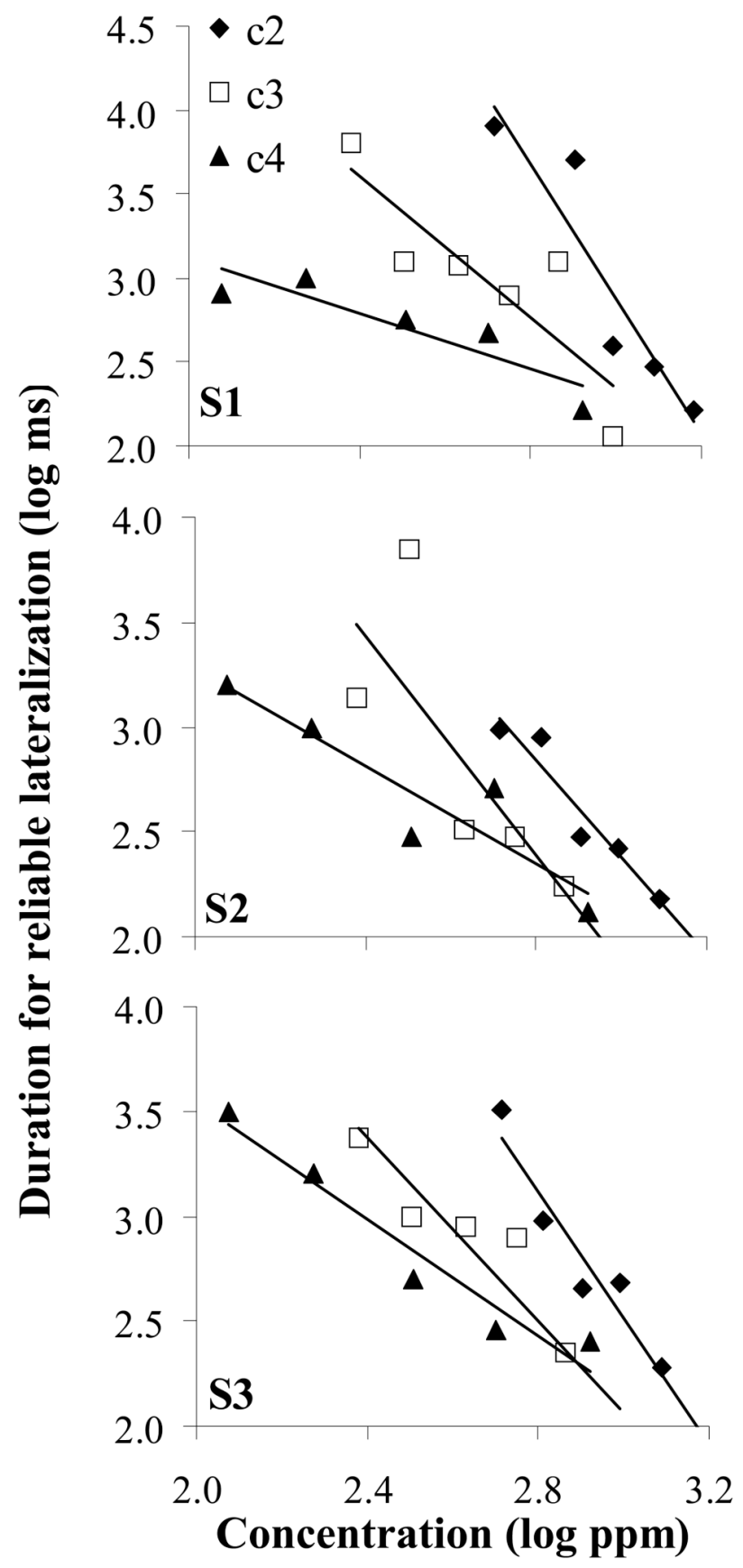

Fig. 2.

Threshold stimulus-duration (log ms) vs. concentration (log ppm) for ethyl propionate (filled diamonds), propyl propionate (open squares), and butyl propionate (filled triangles). Lines represent linear functions fit to data using least-squares regression. The three panels depict data for three individual subjects selected at random from the 10 subjects who received all three chemical stimuli (arbitrarily labeled as S1, S2, and S3). 

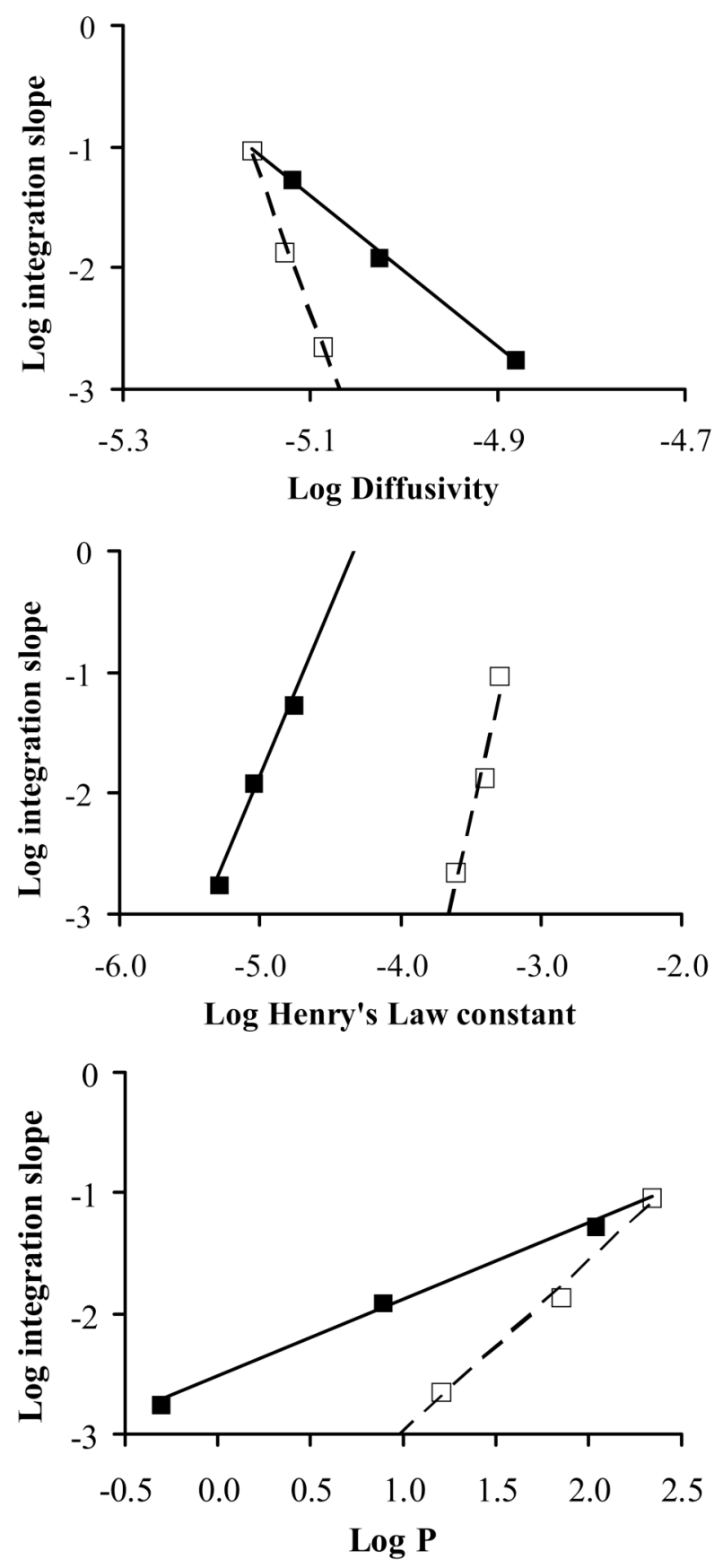

Fig. 3.

Slope of the integration function (Y-axis, as in Figure 1) vs. the three molecular parameters related to solubility and diffusion (X-axes). Top panel: Log of diffusivity; values calculated using the Wilke-Chang equation (Welty et al., 1976). Middle panel: Log of Henry's law constant; published values (Howard et al., 1997). Bottom panel: log octanol/water partition coefficient (Log P); published values (Hansch et al., 1995;Meylan and Howard, 1995). Higher values of diffusivity indicate more rapid diffusion, higher values of Henry's law constant indicate a stronger tendency for molecules to partition into water from air, and higher values of $\log \mathrm{P}$ indicate higher solubility in lipids. Higher values of integration slope indicate better integration. Data for three aliphatic n-alcohols (from Wise et al., 2007, open squares) and the 
three aliphatic n-propionates (filled triangles) are shown. The lines represent the best linear fit (least-squares regression) for the alcohols (dashed) and propionates (solid) separately. 


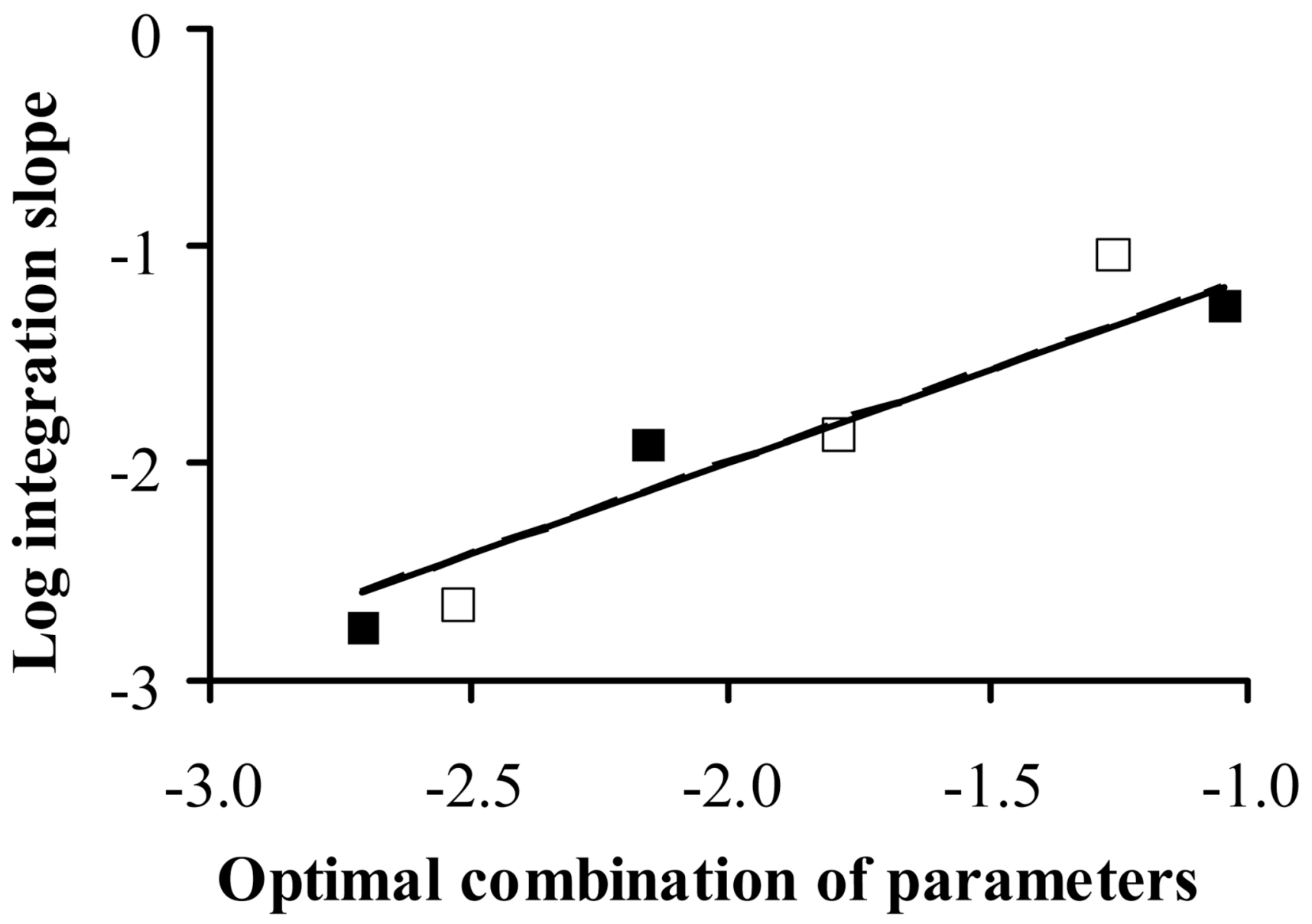

Fig. 4.

Integration slope (Y-axis) vs. an optimal combination of log diffusivity, log Henry's law constant, and $\log \mathrm{P}$ (x-axis). The combination of the three molecular parameters that best predicted actual integration slopes was determined by least-squares multiple regression. Open squares for alcohols, filled triangle for propionates. Separate regression lines were calculated for the alcohols and propionates, but the regression lines for the two chemical families coincide. 


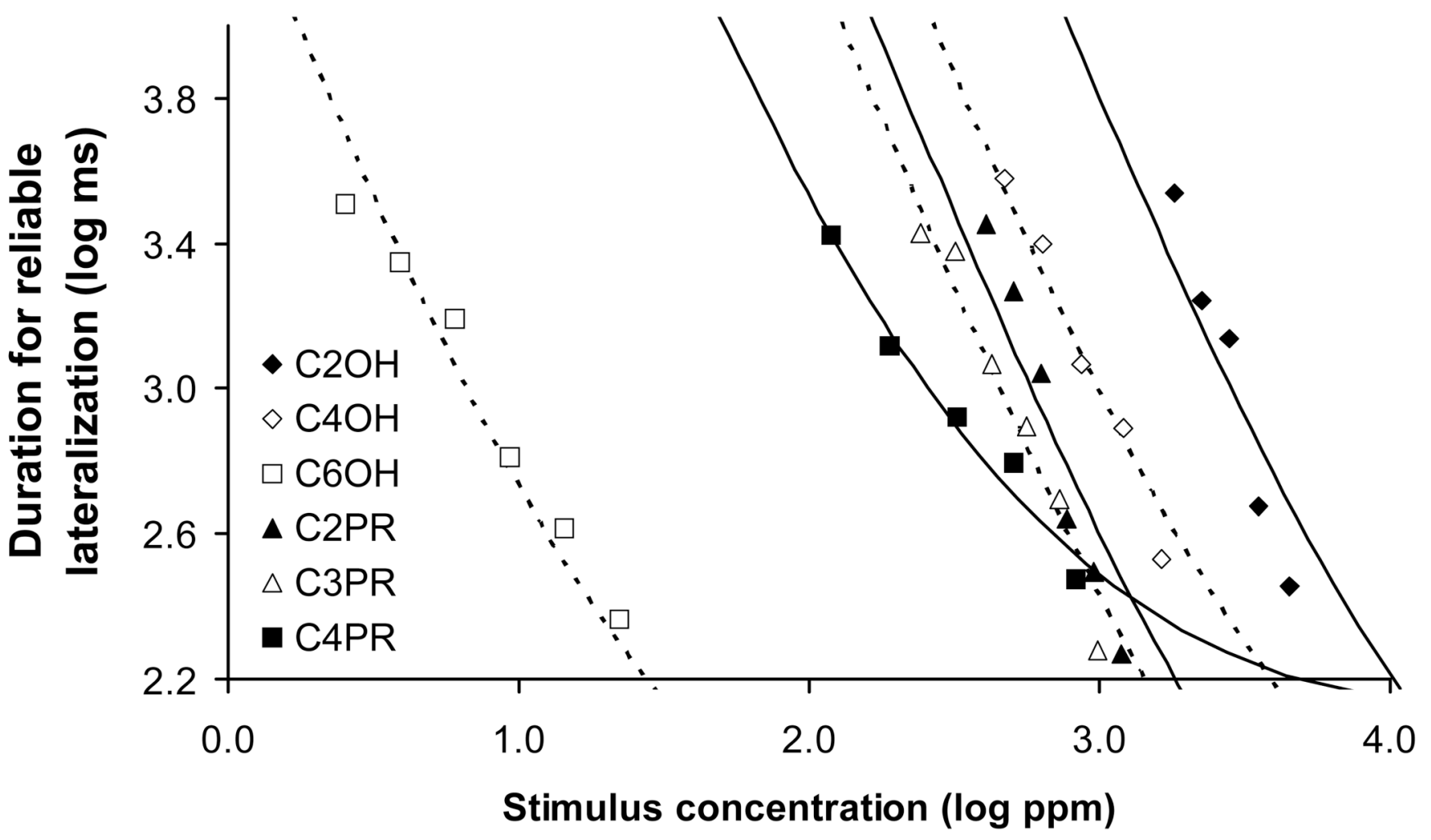

Fig. 5.

Stimulus-duration (log ms) required for reliable lateralization (Y-axis) vs. log stimulus concentration ( $\log \mathrm{ppm}, \mathrm{X}$-axis). Filled and open symbols alternate from left to right for clarity. Functions fit to the data represent least squares fits of a diffusive transport model (see Appendix). Dashed and solid lines alternate for the sake of clarity. $\mathrm{CnOH}=$ alcohols; $\mathrm{CnPR}=$ propionates. 
Table 1

Correlation or multiple correlation coefficients for combinations of up to three parameters

\begin{tabular}{llll}
\hline Parameter & $\mathbf{r}$ & $\mathbf{r}^{2}$ & significance \\
\hline $\log \mathrm{H}$ & -0.29 & 0.08 & $\mathrm{NS}$ \\
$\log \mathrm{D}$ & 0.74 & 0.55 & $\mathrm{NS}$ \\
$\log \mathrm{P}$ & 0.83 & 0.69 & $\mathrm{p}<.05$ \\
$\log \mathrm{D}, \log \mathrm{H}$ & 0.85 & 0.73 & $\mathrm{NS}$ \\
$\log \mathrm{H}, \log \mathrm{P}$ & 0.93 & 0.86 & $\mathrm{NS}$ \\
$\log \mathrm{D}, \log \mathrm{P}$ & 0.95 & 0.90 & $\mathrm{p}<.04$ \\
$\log \mathrm{H}, \log \mathrm{P}, \log \mathrm{D}$ & 0.96 & 0.92 & $\mathrm{NS}$ \\
\hline
\end{tabular}

LogH - Log of Henry's law constant; LogD -Log of diffusivity; LogP - log octanol/water partition coefficient; 584 NS - not significant. 